\title{
Abkürzungen und Siglen
}

Es gelten die Abkürzungen und Siglen des Deutschen Archäologischen Instituts. Für epigraphische Corpora werden die in AE 2014, 753-767 genannten Abkürzungen gebraucht. Zusätzlich werden folgende Siglen verwendet:

AuE

Baladié, Péloponnèse

BCD Peloponnesos

Beekes, EDG

Bourke, Elis

Burkert, HN

Burkert, Religion²

Calame, Choruses

Chantraine, DELG

Cole, Landscapes

Deubner, Feste

DNO

Donati, Agora

Graf, NK

Heiden - Rohn, Triphylien

IACP
A. D. Rizakis (Hrsg.), Achaia und Elis in der Antike. Akten des 1. Interna-

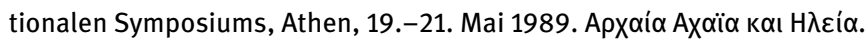

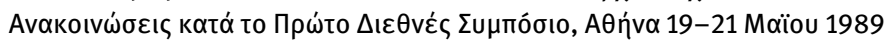
(Athen 1991)

R. Baladié, La Péloponnèse de Strabon: étude de géographie historique (Paris 1980)

Coins of Peloponnesos: The BCD Collection. LHS Numismatics. Auktionskatalog Zürich 8.-9. Mai 2006 (Zürich 2006)

R. S. P. Beekes, Etymological Dictionary of Greek I-II (Leiden 2010)

G. Bourke, Elis: Internal Politics and External Policy in Ancient Greece (London 2017)

W. Burkert, Homo Necans. Interpretationen altgriechischer Opferriten und Mythen ${ }^{2}$ (Berlin 1997)

W. Burkert, Griechische Religion der archaischen und klassischen Epoche ${ }^{2}$ (Stuttgart 2011)

C. Calame, Choruses of Young Women in Ancient Greece (Lanham, Md. 1997)

H. Chantraine, Dictionnaire étymologique de la langue greque (Paris 1968-1980)

S. G. Cole, Landscapes, Gender, and Ritual Space (Berkeley 2004)

L. Deubner, Attische Feste (Berlin 1932)

S. Kansteiner - K. Hallof - L. Lehmann - B. Seidensticker - K. Stemmer (Hrsg.), Der Neue Overbeck. Die antiken Schriftquellen zu den bildenden Künsten der Griechen I-V (Berlin 2014)

J. C. Donati, Towards an Agora: The Spatial and Architectural Development of Greek Commercial and Civic Space in the Peloponnes (Diss. New York University 2010)

F. Graf, Nordionische Kulte. Religionsgeschichtliche und epigraphische Untersuchungen zu den Kulten von Chios, Erythrai, Klazomenai und Phokaia (Rom 1985)

J. Heiden - C. Rohn, Die antike Landschaft Triphylien. Siedlungstopographischer Wandel und Repräsentationsmöglichkeiten im Hellenismus, in: A. Matthaei - M. Zimmermann (Hrsg.), Urbane Strukturen und bürgerliche Identität im Hellenismus (Heidelberg 2015) 330-349

M. H. Hansen - T. H. Nielsen (Hrsg.), An Inventory of Archaic and Classical Poleis (Oxford 2004) 
IED

Jacquemin I

Jacquemin II

Jost, Sanctuaires

LGPN

LSAG $^{2}$

LSCG

LSS

Maddoli - Nafissi Saladino

Maddoli - Saladino

Meyer, Wanderungen

Mitsopoulos-Leon, Heiligtümer

Nielsen, Triphylia

Nilsson, Feste

Nilsson, GGR $\left.\right|^{3}$

NIO

Papachatzis

Parker, Polytheism

Pritchett, Campaign

RGVV

Ruggeri, Stati

Sperling, Elis

Taita, Olimpia
S. Minon, Les inscriptions éléennes dialectales $\left(\mathrm{Vl}^{\mathrm{e}}-\|^{\mathrm{e}}\right.$ siècle avant J.-C.) (Genf 2007)

A. Jacquemin in: M. Casevitz - J. Pouilloux - A. Jacquemin (Hrsg.), Pausanias. Description de la Grèce V: Livre V. L’Elide (I) (Paris 1999)

A. Jacquemin in: M. Casevitz - J. Pouilloux - A. Jacquemin (Hrsg.), Pausanias. Description de la Grèce VI: Livre VI. L'Elide (II) (Paris 2002)

M. Jost, Sanctuaires et cultes d'Arcadie, Études péloponnésiennes 9 (Paris 1985)

P. M. Fraser - E. Matthews (Hrsg.), A Lexicon of Greek Personal Names (Oxford 1997-)

L. H. Jeffery, The Local Scripts of Archaic Greece. Revised Edition with a Supplement by A. W. Johnston (Oxford 1990)

F. Sokolowski, Lois sacrées des cités grecques (Paris 1969)

F. Sokolowski, Lois sacrées des cités grecques. Supplément (Paris 1962)

G. Maddoli - M. Nafissi - V. Saladino (Hrsg.), Pausania. Guida della Grecia. Libro VI: L'Elide e Olimpia ${ }^{3}$ (Mailand 2013)

G. Maddoli - V. Saladino (Hrsg.), Pausania. Guida della Grecia. Libro V: L’Elide e Olimpia (Mailand 1995)

E. Meyer, Neue peloponnesische Wanderungen (Bern 1957)

V. Mitsopoulos-Leon, Heiligtümer und Kultstatuen in Elis, in: G. Grabherr - B. Kainrath - A. Larcher - B. Welte (Hrsg.), Vis imaginum. Festschrift Elisabeth Walde (Innsbruck 2005) 258-268

T. H. Nielsen, Triphylia: An Experiment in Ethnic Construction and Political Organisation, in: T. H. Nielsen (Hrsg.), Yet More Studies in the Ancient Greek Polis, Historia Einzelschriften 108 (Stuttgart 1997) 129-162

M. P. Nilsson, Griechische Feste von religiöser Bedeutung mit Ausschluss der attischen (Leipzig 1906)

M. P. Nilsson, Geschichte der griechischen Religion I. Die Religion Griechenlands bis auf die griechische Weltherrschaft, HAW 5, 2 3 (München 1967)

P. Siewert - H. Taeuber (Hrsg.), Neue Inschriften von Olympia, Tyche Suppl. 7 (Wien 2013)

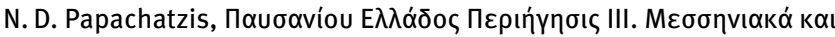

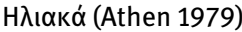

R. Parker, Polytheism and Society at Athens (Oxford 2005)

W. K. Pritchett, Philip's Campaign in the Peloponnesos in 219/8 B.C., in: W. K. Pritchett, Studies in Ancient Topography VI (Berkeley 1989) 1-78

Religionsgeschichtliche Versuche und Vorarbeiten

C. Ruggeri, Gli stati intorno a Olimpia: storia e costituzione dell'Elide e degli stati formati dai perieci elei (400-362 a.C.), Historia Einzelschriften 170 (Stuttgart 2004)

J. Sperling, Explorations in Elis, 1939, AJA 46, 1942, 77-89

J. Taita, Olimpia e il suo vicinato in epoca arcaica (Mailand 2007) 
Taita, Verkehrsverbindungen

Tritsch, Agora

Yalouris, 0ঠпyós
J. Taita, Olympias Verkehrsverbindungen zum Meer: Landungsplätze bei Pheia und am Alpheios, in: H. Kyrieleis, OlBer 13 (Tübingen 2013) 342-396

F. Tritsch, Die Agora von Elis und die altgriechische Agora, ÖJh 27, 1932, 64-105

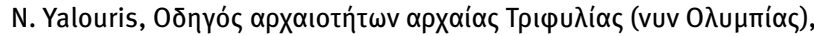

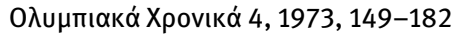


